\title{
A MODIFIED INTERVENTION MODEL FOR GROSS DOMESTIC PRODUCT VARIABLE (AGRICULTURE)
}

\author{
I. A. IWOK AND N. NDUBUISI \\ (Received 16 May 2016; Revision Accepted 16 June 2016)
}

\begin{abstract}
An intervention analysis of agricultural product was conducted. To obtain stationarity, a regular and seasonal differencing was applied to the series. Intervention point of the series was identified in the year 1981. Thus, the pre-intervention period was modeled using the Box and Jenkins (1970) ARIMA methods. Using Akaike Information Criteria (AIC), the pre and post -intervention periods were identified to be Seasonal ARIMA $(0,1,0)(1,0,0)_{4}$ and Seasonal ARIMA $(0,1,1)(2,0,0)_{2}$. On the application of the usual Transfer Function Method, the intervention model was found to be inadequate. To obtain adequacy, a modified approach involving variable reduction was used. The new approach was found to give adequate fit to the series. The model was then used to generate forecast for the next 24 time points.
\end{abstract}

KEYWORDS: Statinarity, Seasonal autoregressive integrated moving average models, Intervention model and Transfer function.

\section{INTRODUCTION}

The economy of any nation largely depends on some economic variables such as building and construction, wholesale and retail, agriculture, industry and services. Traditionally, every country is interested in its economy either by making it stronger or by stabilizing it for the benefit of its citizens. For one to better understand the health of any economy, reference on the past data is needed for such economic history. Hence, the work of most researchers have been recorded and documented for the purpose of reference and historical reasons. In other that data or observations be made relevant in determination and prediction of the future, it becomes necessary that regard is made to time. The method one can use in analyzing such series is "time series" (Box and Jenkins, 1976).

Time series analysis investigates sequence of observations on a variable that have been measured at regularly recurring time points.

The analysis of time series is based on the assumption that successive values in the data file represent consecutive measurements taken at equally time intervals. Time series are most times influenced by different external occurrences such as major work stoppages, technological changes, corporate, economic policy initiatives, etc. These external occurrences are commonly known as interventions. When we understand these interventions, one may try to evaluate the effect of these occurrences and can even link these interventions into our time series model in order to improve parameter estimates or forecasts.

However, if a time series can be subjugated to an intervention over a period of time, there exist two distinct component (parts) in an intervention model: the deterministic part [pre intervention period] and the response(s) [post intervention period]. The purpose for its modeling strategy is to obtain reasonable representations for both distinct components and iterate to an adequate model based on intermediate estimations, diagnostic checks, and interpretation of the model.

It is sometimes difficult to identify a model for the response term since it is affected by the overall series effects. One strategy is to use either of the series to model depending which portion of the residual " $N_{t}$ " is provided in the analysis.

Alternatively, one can also model with the generalized series by comparing the distinct periods. A "composite" choice of model can then be obtained during the process of checking for adequacy of the model based on possible changes made, external effects and on the series residual.

This study examines the intervention effect on the gross domestic product (GDP) variable (Agriculture) in Nigeria.

\subsection{LITERATURE REVIEW}

According to Oduwe (2014), Nigeria is basically an agricultural society even if she relies on the oil industry

I. A. Iwok, Department of Mathematics/Statistics, University of Port-Harcourt, P.M.B.5323, Port-Harcourt, Rivers State, Nigeria.

N. Ndubuisi, Department of Mathematics/Statistics, University of Port-Harcourt, P.M.B.5323, Port-Harcourt, Rivers State, Nigeria. 
for its revenues. $70 \%$ of its population bespeak on agricultural production at an affluence level, though its holdings are small and scattered. It added $41 \%$ to the nation's (GDP) since 1999. The quota to the nation's gross domestic product shows a gradual decrease of $24.7 \%$ from its quota of $65.8 \%$ to the gross domestic product in the 1980's. Though such slip off may not continue as the Nigerian Government is interested in diversifying its economy as a result of less contributions in oil revenues. However, as the economy improves, the size of agricultural input will also improve.

The nation's climate condition allows it to produce varieties of food and cash crops ranging from the staple food crops to variety of fruits and vegetables. Nigeria is a major export nation in the 1960s through 1970s until the inception of petroleum in late 70 s and early 80 s. Amongst its export locations are the United States, France, Britain and Germany.(Ayodele and Falokun, 2003)

Some of the important portion of the agricultural sector includes fishing, poultry, cattle herding etc., which added significantly to the gross domestic product in the 1980s.

Adeboye (2002), in his views on agricultural trade and industrialization process, posits that those who win are those who trade in goods and services characterized by increasing returns. The pace and structure of agriculture are usually dictated by the winners. While in the past, agricultural trade and industrial product were dictated by colonialism, it is driven by more subtle ideology propagated by the international financial institutions and the World Trade Organization (WTO).

According to Iwugwu (2009), industry and agriculture is a sin qua non for sustainable economic growth in Nigeria and it is what the present regime needs to achieve in its transformation agenda since they are the most important and major sectors of the country's economy. Most of the studies on agriculture and economic growth in Nigeria lack essential empirical content. This study is carried out to fill this vacuum.

Imhanlahimhin (2000), in his amfitop book assesses the impact of agriculture on Nigeria economy, given the fact that it is an important sector on the economy. He stresses that agricultural trade has contributed greatly to the Nigerian economy but the revenues derived, have not been properly managed or used considering the fact that there are other sectors of the economy. He continued that the excess money made from other sectors can be invested in agriculture so as to get a diversified economy and to increase the GDP of the economy, which is an economic tool that account for the total number of goods and services produced within an economy in a fiscal year.

Due to the fall in oil prices, most sectors of the Nigeria economy have experienced decline in its output, thereby causing the country to fall back to the old surviving sector (agriculture). The present growing interest in Agricultural sector in relation to the past is therefore of paramount importance to most researchers.
This can best be studied by some techniques that can explain previous, rising and the present behavior of the variable (agriculture). To achieve a purpose, such techniques should be capable of providing an insight into the future, by predicting future values of such variable. It is in this light, that this research emerged.

Intervention analysis is one of such techniques, and it refers to the analysis of the mean level of a series that changes after an intervention when it is assumed that the ARIMA structure of the series $X_{t}$ holds both before and after an intervention. It can be referred to as impact analysis. A researcher can assess the response in a series to a discrete event or intervention input (Makridakis and Wheelright, 1997). These events or interventions are often unusual or singular. The intervention input may be a scandal, war, embargo, strike, or price change etc. (Pack, 1987). The response series may be a popularity rating, a gross domestic product, industrial productivity index, or a level of sales.

According to Balkin and Ord (2001); the standard time series (STS) approach to intervention analysis is based on ARIMA models. In the ARIMA framework, nonstationarity needs transformation prior to the analysis. The usual approach is to transform from non-stationary series to a stationary series and then to fit the distinct appropriate autoregressive moving average (ARMA) model to structure the remaining serial correlation in the series. Structural time series (STS) methods provide an alternative approach to the modeling of interventions (Harvey, 1989). However, structural time series models have been applied in other policies and intervention analysis applications which yielded good results (Harvey and Durbin, 1986; Harvey, 1996).Virtually every field of study has demonstrated the importance and relevancy of intervention analysis approach especially in its ability to enhance forecast base on relating different series.

Iwueze and Biu (2011) applied the effect of intervention analysis to oil and gas production series in Niger Delta region of Nigeria. In this study the effect of an intervention on oil and gas production series was studied using appropriate procedure of Box and Tiao (1976) on intervention analysis, where $\mathrm{AR}(3)$ model with a constant was built for the period prior[pre-intervention] sample and an intervention period model was identified for the period of the data set. The study revealed that monthly production of oil and gas in the region was affected by an external event called Militancy and contributed to reduction of oil and gas product which is affecting the Nation's economy up till date.

Yoonshinnakstad (2006) in his thesis for the Master of Environmental and Development Economics using Intervention Time Series, analyzed GDP's from USA, Euro-area, GBR, Sweden and Japan. From his thesis, he found out that constraining the trend to be stochastic varies the outcomes of the time series. However, he stressed that the stochastic trend should depend on variables, lengths of series, and the purpose of the study. If the trend is so stochastic that it matches almost to the actual series, the cycle will have very small 
disturbance variance. As a result, the model's forecast will be poor. If the trend is not stochastic such as a deterministic trend, the model may forecast well but shows very poor performance on the actual series. The use of interventions affects greatly on the trend's flexibility. With and without interventions give different trend and cycles. However, it was found that for the GDP time series, it is sometimes difficult to identify the background of an intervention.

Tinbergen institute (2003) carried out a work using intervention time series to analyze crime rate and sentence reform in Virginia, Tinbergen institute accessed the impact on crime rate and sentence reform using various time series approach, they observed that the effect of intervention can be estimated using regression model, ARIMA model and structural time series models. In all three different models, the intervention is introduced as a step type of intervention. The results however do not lead to conclusive evidence and therefore more elaborate models were considered.

Camargo and Daquis (2012) used transfer function and intervention models to investigate the dynamics of Brazilian inflationary process between January1980 to December 1993.This was achieved with the following major determinant under consideration: monetary base, wages, federal debt, rate of interest and rate of exchange. The effects of these exogenous variables showed that if the exogenous variable and intervention variables are brought under control, same goes for the inflationary process as well.

As stated earlier, this work seeks to address the time dependence of the agricultural produce in Nigeria, with a view of providing a simplified form of intervention techniques that is capable of providing an insight into the future, by predicting future values of the variable. 


\subsection{METHODOLOGY}

\subsection{Stationarity}

A time series process $\left\{X_{t}\right\}$ is said to be stationary if for a given time points $t_{1}, t_{2}, \ldots, t_{n}$ and any $k$, all the joint moments up to order 2 of $\left\{X_{t_{1}}, X_{t_{2}}, \ldots, X_{t_{n}}\right\}$ exist and is equal to the corresponding joint moments up to order 2 of $\left\{X_{t_{1+k}}, X_{t_{2+k}}, \ldots, X_{t_{n+k}}\right\}$.

\subsection{Autoregressive Model}

The generalized $p^{\text {th }}$ order Autoregressive AR series is given by

$$
x_{t}=\phi_{1} x_{t-1}+\phi_{2} x_{t-2}+\cdots+\phi_{p} x_{t-p}+\varepsilon_{t}
$$

This implies,

$$
\begin{aligned}
& \quad\left(1-\phi_{1} B-\phi_{2} B^{2}-\cdots-\phi_{p} B^{p}\right) x_{t}=\varepsilon_{t} \\
& \text { or } \quad \phi(B) x_{t}=\varepsilon_{t}
\end{aligned}
$$

where;

$$
\phi(B)=\left(1-\phi_{1} B-\phi_{2} B^{2}-\cdots-\phi_{p} B^{p}\right)
$$

\subsection{Moving Average Model}

In terms of deviation from the mean, a series $X_{t}$ is an MA(q) process if it is linearly represented as:

$$
x_{t}=\varepsilon_{t}-\theta_{1} \varepsilon_{t-1}-\theta_{2} \varepsilon_{t-2}-\cdots-\theta_{q} \varepsilon_{t-q}
$$

or

$$
x_{t}=\theta(B) \varepsilon_{t}
$$

where

$$
\theta(B)=\left(1-\theta_{1} B-\theta_{2} B^{2}-\ldots-\theta_{q} B^{q}\right),
$$

$\varepsilon_{\mathrm{t}}$ is white noise and $\theta_{1}, \theta_{2}, \ldots, \theta_{q}$ are constants

\subsection{Mixed Autoregressive-Moving Average (ARMA) Model}

The combination of both AR $(p)$ and MA $(q)$ model result in a much flexible model called the ARMA $(p, q)$ and is expressed as:

$x_{t}-\phi_{1} x_{t-1}-\phi_{2} x_{t-2}-\cdots-\phi_{p} x_{t-p}=\varepsilon_{t}-\varepsilon_{t-1}-\theta_{2} \varepsilon_{t-2}-\cdots-\theta_{q} \varepsilon_{t-q}$

or

$$
\phi(B) x_{t}=\theta(B) \varepsilon_{t}
$$

This is equivalent to

$$
\begin{aligned}
x_{t} & =\phi^{-1}(B) \theta(B) \varepsilon_{t} \\
& =\frac{\theta(B)}{(B)} \varepsilon_{t}=\frac{1-\theta_{1} B-\cdots-\theta_{q} B^{q}}{1-\phi_{1} B-\cdots-\phi_{p} B^{P}} \varepsilon_{t}
\end{aligned}
$$

3.5 Seasonal Autoregressive Integrated moving average (SARIMA) Model The general SARIMA model [SARIMA $\left.(p, d, q)(P, D, Q)_{s}\right]$ is given by

$$
\phi_{p}(B) \phi_{p}\left(B^{s}\right) \nabla^{\mathrm{d}} \nabla_{\mathrm{s}}^{\mathrm{D}} \mathrm{z}_{\mathrm{t}}=\theta_{\mathrm{q}}(\mathrm{B}) \theta_{\mathrm{Q}}\left(\mathrm{B}^{\mathrm{s}}\right) \varepsilon_{\mathrm{t}}
$$


where

$$
\begin{aligned}
& \phi(B)=1-\phi_{1} B-\phi_{2} B^{2}-\cdots \phi_{p} B^{p}, \Phi(B)=1-\Phi_{1}, s B^{s}-\Phi_{2}, s B^{2 s}-\cdots-\Phi_{p}, s B^{p}, \\
& \nabla^{d}=1-B-B^{2}-\cdots-B^{d}, \nabla_{s}^{D}=1-B^{s}-B^{2 s}-\cdots-B^{2 D}, \\
& \theta(B)=1-\theta_{1} B-\theta_{2} B^{2}-\cdots-\theta_{q} B^{q}, \\
& \Theta\left(B^{s}\right)=1-\Theta_{1}, s B^{s}-\Theta_{2}, s B^{2 s}-\cdots-\Theta_{Q}, s B^{Q s}
\end{aligned}
$$

$Z_{t}$ is the time series at period $t, \varepsilon_{t}$ is the white noise process,

$B$ is the backshift operator, $S$ is the season, $p$ is the order of Autoregressive part,

$P$ is the order Seasonal autoregressive part, $d$ is the order of non seasonal differencing, $D$ is the order of seasonal difference, $q$ is order of moving average part, $Q$ is the order of seasonal moving average part.

\subsection{Model Selection Criteria}

The criteria used in selecting the model is the Akaike's Information Criterion (AIC) given by

$$
\mathrm{AIC}=N \log \hat{\sigma}^{2}+2 k
$$

where

$k \quad$ is the number of model parameters,

$N$ is the number of data point used in the estimation procedure and

$\hat{\sigma}^{2}$ is the estimated residual variance.

The order of the model is the lags at which the AIC is minimum.

\subsection{The intervention model}

The ARIMA model $X_{t}$ without intervention is:

$$
X_{t}-\mu=\frac{\theta(\mathbf{B})}{\phi(B)} \omega_{t}
$$

where $X_{t}$ is the series observed, $\omega_{t}$ is the error term.

$\theta(B)$ and $\phi(B)$ is the MA and AR series (polynomial) respectively.

Let $Z_{t}=\Delta t$ attributed to the intervention; and by definition $Z_{t}=0$ before the intervention time. The value of $Z_{t}$ can either be zero or one after $T$ (the intervention point).

The generalize model with the intervention effect is:

$$
x_{t}=z_{t}+\frac{\theta(\mathrm{B})}{\phi(B)} w t
$$

\subsection{Transfer Functions and the Intervention Model}

Suppose we denote the $t^{\text {th }}$ value of the input and out-put non-stationary series by $X_{t}$ and $Y_{t}$.

Let $y_{t}=\Delta Y_{t}$ and $x_{t}=\Delta X_{t}$ be the stationary series produced by the above transformation. Applying the Box and Jenkins (1976) model identification method, we express an ARIMA model of the form:

$\begin{aligned} \phi_{p}(B) \eta_{t} & =\theta_{q}(B) e_{t}\end{aligned}$

According to Box and Tiao (1976) the Transfer function model is

$$
y_{t}=\mu+\frac{\omega(\mathrm{B})}{\delta(B)} B^{b} x_{t}+\eta_{t}
$$


where,

$\eta_{t}$ represents the error, $b$ is the number of periods, $\omega(B)=\omega_{0}-\omega_{1} B-\omega_{2} B^{2}-\ldots-\omega_{s} B^{s}$,

$\delta(B)=1-\delta_{1} B-\delta_{2} B^{2}-\ldots-\delta_{r} B^{r}, \mu$ is a constant term. Then

$$
y_{t}=\mu+\frac{\omega(\mathrm{B})}{\delta(B)} X_{t-b}+\frac{\phi_{p}(B)}{\theta_{q}(B)} e_{t}
$$

Hence,

$$
y_{t}=\mu+\frac{\left(1-w_{1} B-w_{2} B^{2}-\cdots-w_{s} B^{s}\right) X_{t-b}}{\left(1-\delta_{1} B-\delta_{2} B^{2}-\cdots-\delta_{r} B^{r}\right)}+\frac{\left(1-\theta_{1} B-\theta_{2} B^{2}-\cdots-\theta_{q} B^{q}\right)}{\left(1-\phi_{1} B-\Phi_{2} B^{2}-\cdots-\theta_{p} B^{p}\right)} e_{t}
$$

For an intervention process, we may replace $\eta_{t}$ with $y_{t}$ in equation (7) to obtain the Autoregressive Integrated Moving Average model as:

$$
\begin{array}{ll} 
& \phi_{p}(B) \nabla^{d} y_{t}=\theta_{q}(B) e_{t} \\
\Rightarrow & y_{t}=\frac{\left(1-\theta_{1} B-\theta_{2} B^{2}-\cdots-\theta_{q} B^{q}\right)}{(1-B)\left(1-\phi_{1} B-\phi_{2} B^{2}-\cdots-\theta_{p} B^{p}\right)} e_{t}
\end{array}
$$

By hypothesizing the effect of the intervention on $y_{t}$, we obtain:

$$
y_{t}=S_{t}+\frac{\left(1-\theta_{1} B-\theta_{2} B^{2}-\cdots-\theta_{q} B^{q}\right)}{(1-B)\left(1-\Phi_{1} B-\Phi_{2} B^{2}-\cdots-\theta_{y} B^{p}\right)} e_{t}
$$

where:

$$
S_{t}=\left\{\begin{array}{l}
0 \text { when } t<T \\
1 \text { when } t \geq T
\end{array}\right.
$$

$S_{t}$ is a step function and $\mathrm{T}$ is the time where the intervention starts.

To estimate the parameters of the model we can write equation (14) as a transfer function model as follows:

$$
\begin{gathered}
(1-B)\left(1-\phi_{1} B-\Phi_{2} B^{2}-\cdots-\theta_{p} B^{p}\right) y_{t}=(1-B)\left(1-\phi_{1} B-\phi_{2} B^{2}-\cdots-\theta_{p} B^{p}\right) S_{t} \\
+\left(1-\theta_{1} B-\theta_{2} B^{2}-\cdots-\theta_{q} B^{q}\right) e_{t}
\end{gathered}
$$

We let

$$
\begin{aligned}
Z_{t} & =Z_{t}^{(x]}+\left(1-\theta_{1} B-\theta_{2} B^{2}-\cdots-\theta_{q} B^{q}\right) e_{t} \\
& =\frac{\omega(\mathrm{B})}{\delta(B)} B^{b} z_{t}^{(x)}+\left(1-\theta_{1} B-\theta_{2} B^{2}-\cdots-\theta_{q} B^{q}\right) e_{t}
\end{aligned}
$$

where:

$Z_{t}=(1-B)\left(1-\phi_{1} B-\phi_{2} B^{2}-\cdots-\theta_{p} B^{p}\right) y_{t}$,

$Z_{t}^{(x)}=(1-B)\left(1-\phi_{1} B-\phi_{2} B^{2}-\cdots-\theta_{p} B^{p}\right) S_{t}, \omega(B)=1, \delta(B)=1$ and $b=0$ :

which by this arrangement is a transfer function model.

Rather than expressing equation (12) as a transfer function, we can express it directly as:

$\left(1-\phi_{1} B-\phi_{2} B^{2}-\cdots-\phi_{p} B^{p}-B+\phi_{1} B^{2}+\phi_{2} B^{3}-\cdots-\phi_{2} B^{2 p}\right) Y_{t}=$

$\left(1-\phi_{1} B-\phi_{2} B^{2}-\cdots-\phi_{p} B^{p}-B+\phi_{1} B^{2}+\phi_{2} B^{3}-\cdots-\phi_{2} B^{2 p}\right) S_{t}$

$+\left(1-\theta_{1} B-\theta_{2} B^{2}-\cdots-\theta_{q} B^{q}\right) e_{t}$ 


$$
\begin{aligned}
& \Rightarrow Y_{t}-\phi_{1} Y_{t-1}-\phi_{2} Y_{t-2}-\cdots-\phi_{p} Y_{t-p}-Y_{t-1}+\phi_{1} Y_{t-2}+\phi_{2} Y_{t-3}-\cdots-\phi_{p} Y_{t-2 p}= \\
& S_{t}-\phi_{1} S_{t-1}-\phi_{2} S_{t-2}-\cdots-\phi_{p} S_{t-p}-S_{t-1}+\phi_{1} S_{t-2}+\phi_{2} S_{t-3}-\cdots-\Phi_{p} S_{t-2 p}+ \\
& e_{t}-\theta_{1} e_{t-1}-\theta_{2} e_{t-2}-\cdots-\theta_{q} e_{t-q} \quad \cdots \cdots \cdots \cdots \cdots \cdots \cdots \cdots \cdots \cdots \cdots \cdots \cdots \cdots \cdots \cdots \cdots \cdots \cdots \cdots \cdots \cdots \cdots \cdots \cdots \cdots \cdots \cdots \cdots \cdots \cdots \cdots \cdots \cdots \cdots \cdots \cdots \cdots \cdots \cdots \cdots \cdots \cdots \cdots \cdots \cdots \cdots \cdots \cdots \cdots \cdots \cdots \cdots
\end{aligned}
$$

This can be reduced to:

$Y_{t}=P_{1 t}+P_{2 t}+P_{3 t}+e_{t}$

where,

$\boldsymbol{P}_{1 t}=Y_{t}-\phi_{1} Y_{t-1}-\phi_{2} Y_{t-2}-\cdots-\phi_{p} Y_{t-p}-Y_{t-1}+\phi_{1} Y_{t-2}+\phi_{2} Y_{t-3}-\cdots-\phi_{p} Y_{t-2 p}$

$P_{2 t}=S_{t}-\phi_{1} S_{t-1}-\phi_{2} S_{t-2}-\cdots-\phi_{p} S_{t-p}-S_{t-1}+\phi_{1} S_{t-2}+\phi_{2} S_{t-3}-\cdots-\phi_{p} S_{t-2 p}$

$P_{3 t}=e_{t}-\theta_{1} e_{t-1}-\theta_{2} e_{t-2}-\cdots-\theta_{q} e_{t-q}$

are new series.

\subsection{Diagnostic Checks}

This process is accomplished by investigating the autocorrelation function (ACF) and partial autocorrelation function (PACF) plots of the residuals and the use of Q-test. If the appropriate model has been chosen, there will be zero autocorrelation of the residuals. One of the useful tools of testing the model adequacy is the Ljung and Box (1978) Statistic. This is given by

$$
\tilde{Q}(\hat{r})=n(n+2) \sum_{k=1}^{m} \frac{\hat{r}_{k}^{2}}{n-k}
$$

Considering the first $m$ autocorrelations, $n$ is the sample size and $r_{k}$ is the autocorrelation at lag $k$.

Then, under the assumption of model adequacy, $Q$ is approximately distributed as $\chi^{2}$ (Box and Tio, 1976).

On the other hand, if the model is inappropriate, the average values of $Q$ will be inflated.

More formally, the Ljung-Box test can be defined as follows:

$H_{0}$ : The residuals are serially uncorrelated.

$H_{a}$ : The residuals are serially correlated

Under this hypothesis, a series $\left\{\varepsilon_{t}\right\}$ is said to be serially uncorrelated if and only if its autocorrelation function is zero.

\subsection{Forecasts}

When once the fitted model is found to be adequate, forecast can be generated from the model.

\subsection{Data Analysis and Result}

The data used for this work is a secondary data of Quarterly Real Gross Domestic Product, obtained from the
Central Bank of Nigeria (CBN), annual statistical bulletin (2013). The variable of interest is "agriculture" of the Nigerian Gross Domestic Product (GDP). The data runs in years from 1965-2013, with each year starting from January through December.

The plot of the raw data is shown in figure 1 


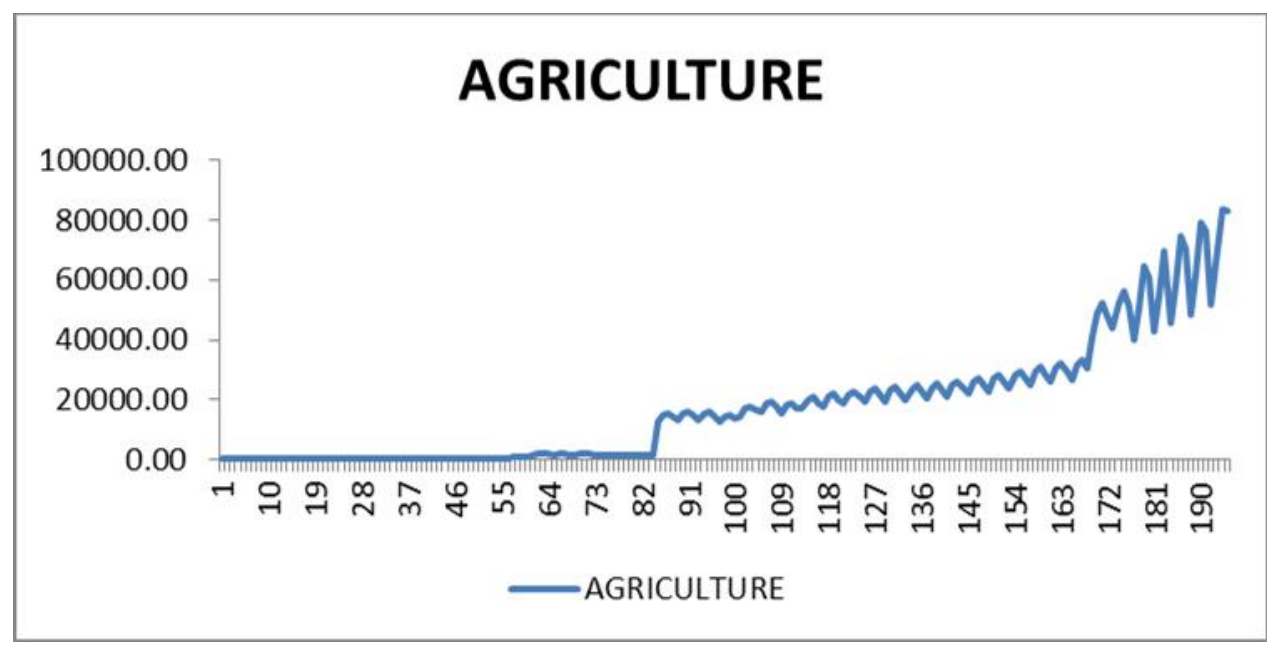

Figure 1: Agriculture sectors current basic prices (Naira Million), 1965 to 2013

Examining figure 1, we noticed a linear change, with abrupt start (strike) in the year 1981. The strike occurred at time, $\mathrm{t}=84$. The impact started at $\mathrm{t}=85$ and is maintained till $t=196$. Thus, there is one intervention point and we have the pre-intervention period (1965 to 1981) and post-intervention period (1981 to 2013).

According to Box and Tiao (1976), analysis of such series can be easily done, in this case Government policy on agriculture.

\subsection{Model identification for Pre and Post intervention Series}

To construct a suitable intervention time series model that would be used to forecast the data on agriculture sector, we employed Box and Jenkins (1978) methods to identify a model for both the pre and postintervention period.

Using the Akaike Information Criteria (AIC), we obtain for the pre-intervention period the selected model that minimizes this criterion to be Seasonal $\operatorname{ARIMA}(0,1,0)(1,0,0)_{4}$. Thus, we have for the preintervention period

$$
\nabla\left(1-\phi_{1,4} \mathrm{~B}^{4}\right) \mathrm{X}_{t}=e_{t}
$$

which gives

$$
\mathrm{X}_{t}=\mathrm{X}_{t-1}+0.3412 \mathrm{X}_{t-4}-0.3412 \mathrm{X}_{t-5}+e_{t}
$$

Similarly, for the post intervention period, we have the best fitted model to be

Seasonal $\operatorname{ARIMA}(0,1,1)(2,0,0)_{2}$. That is,

$$
\nabla\left(1-\phi_{1,2} \mathrm{~B}-\phi_{2,2} \mathrm{~B}^{2}\right) \mathrm{X}_{t}=\left(1-\theta_{1} \mathrm{~B}\right) e_{t}
$$

which gives

$$
\mathrm{X}_{t}=-0.659 \mathrm{X}_{t-1}-0.91 \mathrm{X}_{t-2}-0.49 \mathrm{X}_{t-3}+e_{t}-0.38 e_{t-1}
$$

Where $\nabla=1-B$

\subsection{The Intervention Model}

Using Box and Tiao (1976) method, the identified Seasonal ARIMA model for the(pre-intervention) period, $\left(1965\right.$ - 1981) is Seasonal $\operatorname{ARIMA}(0,1,0)(1,0,0)_{4}$., and post intervention period (1982 - 2013) is Seasonal ARIMA $(0,1,1)(2,0,0)_{2}$

Incorporating the Seasonal ARIMA models in equations 20 and 21 with the intervention components; we have for the pre-intervention period: 


$$
X_{1 t}=\frac{e_{t}}{(1-\mathrm{B})\left(1-\phi_{2,4} \mathrm{~B}^{4}\right)}
$$

and for the post intervention period:

$$
X_{2 t}=\frac{\left(1+\phi_{1} \mathrm{~B}\right)}{(1-\mathrm{B})\left(1-\phi_{1,2} \mathrm{~B}-\phi_{2,2} \mathrm{~B}\right)} e_{t}
$$

combining the two models, we obtain the generalized intervention model:

$$
Y_{t}=S_{t}+\frac{1}{(1-\mathrm{B})\left(1-\phi_{1,4} \mathrm{~B}^{4}\right)}+\frac{\left(1+\phi_{1} \mathrm{~B}\right)}{(1-\mathrm{B})\left(1-\phi_{1,2} \mathrm{~B}-\phi_{2,2} \mathrm{~B}^{2}\right)} e_{t}
$$

where,

$$
\begin{aligned}
& S_{1 t}=\left\{\begin{array}{l}
1: t=\text { January } 1965-\text { December } 1981 \\
0: \text { otherwise }
\end{array}\right. \\
& S_{2 t}=\left\{\begin{array}{l}
1: t \geq \text { December } 1981 \\
0: \text { otherwise }
\end{array}\right.
\end{aligned}
$$

In practice, the intervention component is usually incorporated only in the pre-intervention model for forecasting. Thus, for the pre-intervention period, we had

$$
\begin{array}{ll} 
& \nabla\left(1-\phi_{1,4} \mathrm{~B}^{4}\right) \mathrm{X}_{t}=e_{t} \\
\text { Let } \quad \mathrm{X}_{\mathrm{t}}=\mathrm{Y}_{\mathrm{t}}
\end{array}
$$

Incorporating the intervention component $S_{t}$ into the pre-intervention equation, we have:

$$
Y_{t}=S_{t}+\frac{e_{t}}{(1-\mathrm{B})\left(1-\phi_{14} \mathrm{~B}^{4}\right)}
$$

where,

$$
S_{t}=\left\{\begin{array}{l}
1: t=1965-1981 \\
0: \text { otherwise }
\end{array}\right.
$$

and

the estimated value of $\quad \phi_{1,4}=0.3412$

Equation (22) can further be evaluated as:

$$
\begin{gathered}
Y_{t}\left((1-\mathrm{B})\left(1-\phi_{1,4} \mathrm{~B}^{4}\right)\right)=S_{t}\left((1-\mathrm{B})\left(1-\phi_{1,4} \mathrm{~B}^{4}\right)\right)+e_{t} \\
\left((1-\mathrm{B})\left(1-\phi_{1,4} \mathrm{~B}^{4}\right)\right) Y_{t}=\left((1-\mathrm{B})\left(1-\phi_{1,4} \mathrm{~B}^{4}\right)\right) S_{t}+e_{t}
\end{gathered}
$$

So that,

$$
(1-B)\left(1-0.3412 B^{4}\right) Y_{t}=(1-B)\left(1-0.3412 B^{4}\right) S_{t}+e_{t}
$$

Presenting the above as a transfer function model, we have

$$
\begin{aligned}
Z_{t} & =Z_{t}^{(x)}+e_{t} \\
& =\frac{\omega(B)}{\delta(B)} B^{b} Z_{t}^{(x)}+e_{t}
\end{aligned}
$$


where,

$Z_{t}=(1-B)\left(1-\phi_{1,4} B^{4}\right) X_{t}, Z_{t}^{(x)}=(1-B)\left(1-\phi_{1,4} B^{4}\right) S_{t}$

$\omega(B)=1, \delta(B)=1$,and $b=0$, where $\phi_{1,4}=0.3412$

Under the hypothesis of zero auto correlation, the residual autocorrelation plot (see figure A.1 of appendix A) of fitting the intervention model by the above transfer function approach contains some significant spikes. This is an indication that the model is inadequate. .

\subsection{The Modified Approach}

As stated in equation (18), we boycott the transfer function approach and present equation (22) as:

$X_{t}=S_{t}+\left(\frac{1}{(1-B)(1-0.3412)}\right) e_{t}$

Evaluating the above, we have

$X_{t}(1-B)\left(1-0.3412 B^{4}\right)=(1-B)\left(1-0.3412 B^{4}\right) S_{t}+e_{t}$

$X_{t}-0.3412 X_{t-4}-X_{t-1}+0.3412 X_{t-5}=S_{t}-0.3412 S_{t-4}-S_{t-1}+0.3412 S_{t-5}+e_{t}$

So that

$X_{t}-X_{t-1}-0.3412 X_{t-4}+0.3412 X_{t-5}=S_{t}-S_{t-1}-0.3412 S_{t-4}+0.3412 S_{t-5}+e_{t}$

where $x_{t}=X_{t}-X_{t-1}$, then

$x_{t}-0.3412 X_{t-4}+0.3412 X_{t-5}=S_{t}-S_{t-1}-0.3412 S_{t-4}+0.3412 S_{t-5}+e_{t}$

This implies,

$x_{t}=0.3412 X_{t-4}-0.3412 X_{t-5}+S_{t}-S_{t-1}-0.3412 S_{t-4}+0.3412 S_{t-5}+e_{t}$

Hence, the reduced form can be expressed as:

$x_{t}=P_{1 t}+P_{2 t}+e_{t}$

Where

$P_{1 t}=0.3412 X_{t-4}-0.3412 X_{t-5}$

$P_{2 t}=S_{t}-S_{t-1}-0.3412 S_{t-4}+0.3412 S_{t-5}$

are two independent new series.

$e_{t}$ is assumed to follow a white noise process and $x_{t}=X_{t}-X_{t-1}$

and $x_{t}$ is now dependent on only two series $P_{1 t}$ and $P_{2 t}$.

This can be presented in regression form as

$x_{t}=c_{1} P_{1 t}+c_{2} P_{2 t}+e_{t}$

Where $c_{1}$ and $c_{2}$ are constants and $e_{t}=x_{t}-\left(c_{1} P_{1 t}+c_{2} P_{2 t}\right)$

Analyzing the above equation (30), we obtain

$\hat{x}_{t}=0.36 P_{1 t}+0.42 P_{2 t}$

\subsection{Diagnosis}

To check for adequacy, the following diagnostic checks are applied.

\subsection{Autocorrelation Function for the Residual}

The autocorrelation function plot of the residual term (see figure A.2 of appendix A) shows no significant spikes.

This indicates that the residual follows a white noise process. Hence the model is adequate. 


\subsection{Ljung-Box Q-Test}

Under this test (as described in the methodology), we have the hypothesis:

$H_{0}$ : The residuals are serially uncorrelated.

$H_{a}$ : The residuals are serially correlated

Then, considering the first $\mathrm{m}=25$ autocorrelations, we have

$\mathrm{Q}=196(196+2) \sum_{j=1}^{25} \frac{0.0174}{196}+\frac{0.0038}{195}+\frac{0.0595}{194}+\cdots+\frac{0.0022}{184}=15.6632$

Since $Q<\chi_{0.05, m}^{2}=37.652$; there is not enough evidence to reject $H_{0}$. Hence, by this test, the fitted model is adequate.

\subsection{Forecasts}

Having tested that the modified model fitted to the data is adequate; the next step is to use it to generate forecast. The generated forecasts are displayed in appendix B.

\subsection{DISCUSSION}

Usually, intervention analysis is carried out using transfer function approach. It is observed, however, that obtaining an appropriate intervention model through this method is always tedious. In most cases, it does not give good parameter estimates. To get an appropriate specification, a parsimonious model is required. This work has provided a simple frame work for obtaining models with intervention events by reducing the number of original variables to new ones that can easily be handled.

\section{CONCLUSION}

In most cases, the intervention model (using the transfer function approach) may not give an adequate fit to a data that contains an intervention. The result of this work shows that the modified model (using variable reduction) is much appropriate in addressing such situations of abrupt rise or fall with permanent effect. It also investigates the probabilistic nature of the variables and provides a forecasting tool of future events.

\section{REFERENCES}

Adeboye, I. B., 2002. Forecasting exchange rate between the Ghana Cedi and the US Dollar using time series analysis. African Journal of Basic applied sciences, 3, (6): 255-264.

Ayodele, A. I and Falokun, G., 2003. The Nigerian Economy: Structure Pattern of Development. JODAD publishers. ISBN: 4-542-31134-6.

Box, G. E. P and Tiao, G., 1976. Intervention analysis applications to economic and environmental problems. Journal of the American statistical association, 70, (349): 70-79.
Box, G. E. P and Jenkins, G., 1976. Time Series Analysis, Forecasting and Control, Holden-Day San Francisco.

Camargo, $\mathrm{P}$ and Daquis, H., 2012. Nonparametric transfer function model with localized temporal effect. The Philippine Statistician, 62, (1): 1-14.

Central Bank of Nigeria., 2013. Statistical Bulletin. Retrieved from cenbank.org.

Daniel, W. U., 2010. Lecture note on "Time Series Analysis" for undergraduate student, University of Port Harcourt.

Harvey, A. C., 1989. Forecasting Structural Time Series Models and the Kalman Filter Cambridge, Cambridge University Press. ISBN: 4-2213-10535.

Harvey, A. C., 1996. Intervention analysis with control groups, International Statistical Review64: 313328.

Harvey, A. C and Durbin, J., 1986. The effect of seat belt legislation on British road casualties. A case study in Structural Time Series Modeling, Journal of Royal Statistical Society B 149:189-227.

Imhanlahimhin, J. E., 2000. Development Administration in Less Development Countries. Ibadan: Amfitop Books. ISBN: 2-2212-4133-8.

Iwugwu, O., 2009. The Cluster Concept: Will Nigeria's New Industrial Development Strategy Jumpstart The Country's Industrial Takeoff? Afro Asian Journal of Social Sciences, 2, (24): Quarter IV 2011.

Iwueze, I. S and Biu, O. E., 2011. Application of Intervention Analysis to Oil and Gas Production Series in Niger Delta, Nigeria $\left(35^{\text {th }}\right.$ Annual SPE 
International Technical Conference and Exibition in Abuja, Nigeria. August 1-3, 2011), pp 3-4.

Ljung, G. M and Box, G. E. P., 1978. On a measure of lack of fit in time series models, Biometrika 65, 297-303.

Makridakis, S and Wheelwright, S., 1997. Forecasting: Methods and Applications, 3rd Ed. New York: John Wiley and Sons, pp. 373, 520-542, 553-574.

Oduwe, J. J., 2014. The role of Agriculture in Nigerian Economy. International Journal of Agricultural science, 2, (5): 21-26. ISBN: 3-1314-4252-8.

Park, D., 1987. A Practical Overview of ARIMA Models for Time Series Forecasting.
Wei, W., 2006. Time series analysis; Univariate and Multivariate Models $2^{\text {nd }}$ Ed.New York; Addison Wesley. ISBN: 0-321-32216-9.

Yoon shin Nakstad., 2006. Environmental and Development Economics using Intervention Time Series. Department of Economics, University of Oslo. ISBN: 0-143-24453-7. 


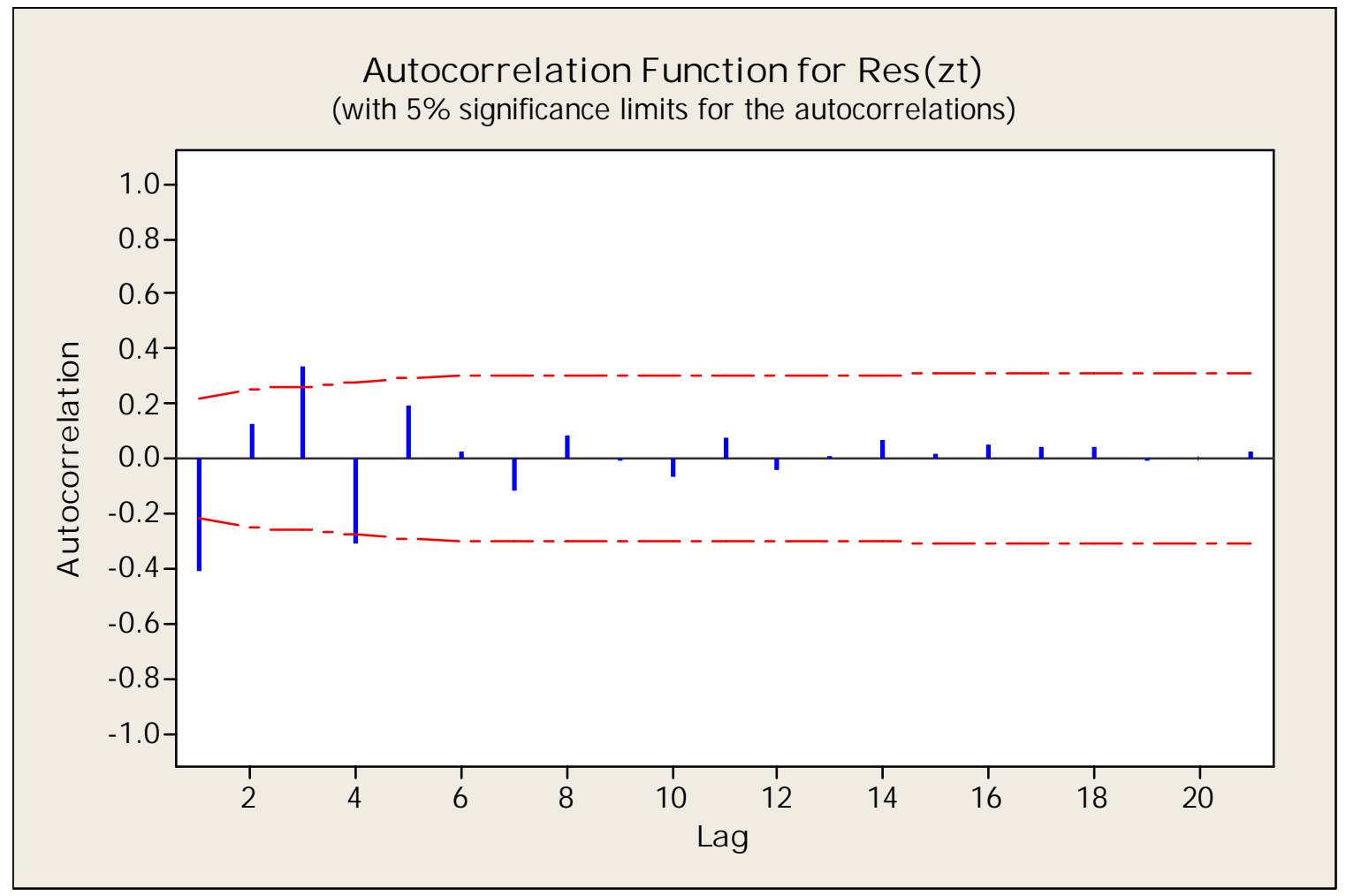

Figure A.1: Residual autocorrelation plot of the intervention (using transfer function approach) model

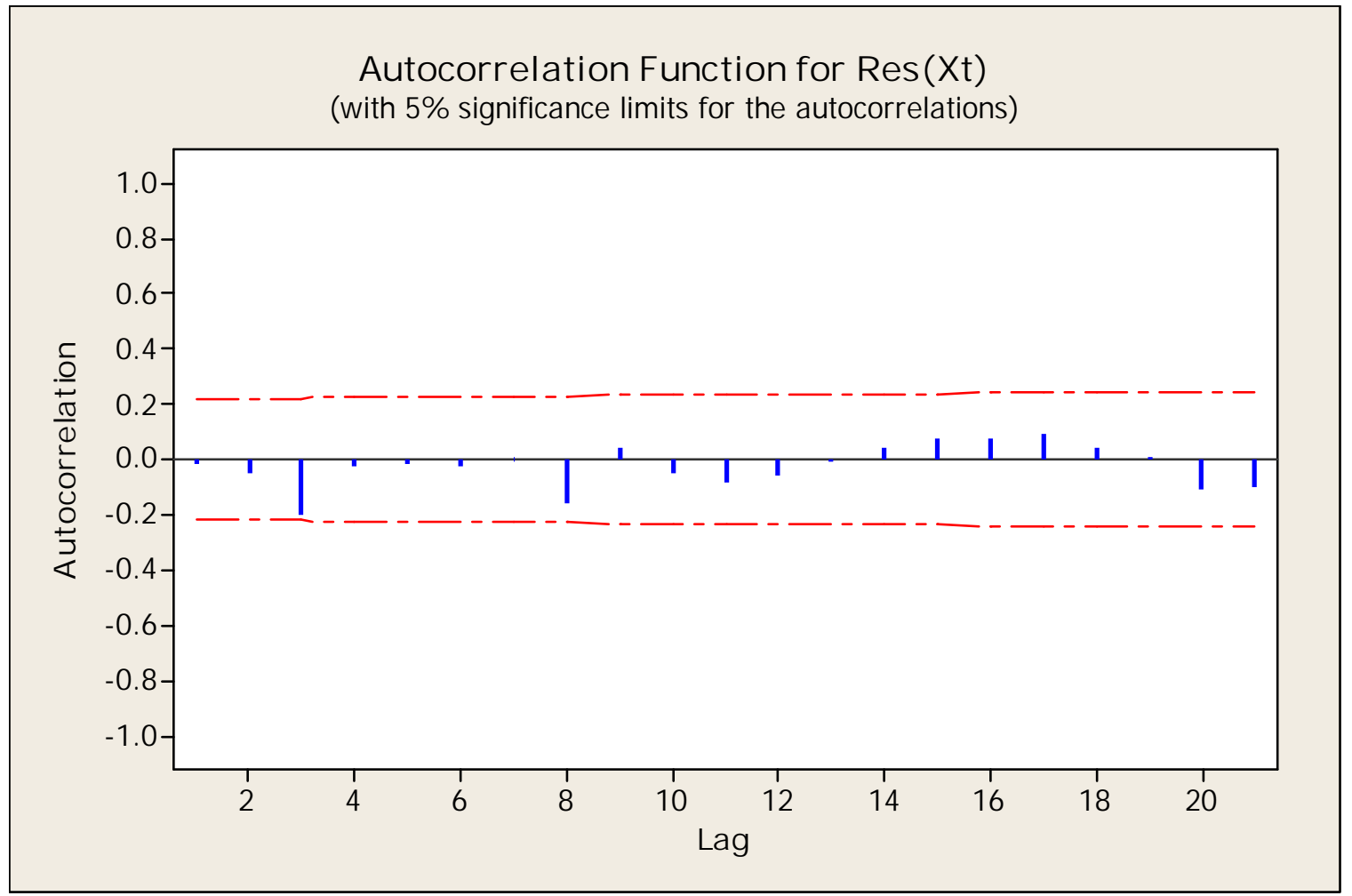

Figure A. 2: Residual autocorrelation plot of the modified model 


\section{APPENDIX B (FORECASTS)}

\begin{tabular}{rrrrr}
\multicolumn{4}{c}{ Forecasts from period 196} \\
\multicolumn{4}{c}{ 95 Percent } \\
Period & \multicolumn{4}{c}{ Limits } \\
197 & 61550 & 55511 & 67589 \\
198 & 68466 & 61225 & 75706 \\
199 & 86992 & 79676 & 94308 \\
200 & 83604 & 76008 & 91200 \\
201 & 65915 & 56204 & 75626 \\
202 & 70917 & 60131 & 81702 \\
203 & 87835 & 76947 & 98722 \\
204 & 84363 & 73176 & 95550 \\
205 & 68995 & 56376 & 81615 \\
206 & 73225 & 59747 & 86704 \\
207 & 88326 & 74732 & 101921 \\
208 & 85195 & 71320 & 99070 \\
209 & 71702 & 56736 & 86668 \\
210 & 75437 & 59766 & 91109 \\
211 & 88851 & 73051 & 104652 \\
212 & 86106 & 70042 & 102171 \\
213 & 74233 & 57286 & 91180 \\
214 & 77570 & 60022 & 95118 \\
215 & 89476 & 71789 & 107163 \\
216 & 87090 & 69149 & 105030 \\
217 & 76641 & 57960 & 95322 \\
218 & 79633 & 60427 & 98839 \\
219 & 90203 & 70849 & 109558 \\
220 & 88138 & 68538 & 107738
\end{tabular}


Appendix C

Quarterly Agriculture Data (Activity Sector)

\begin{tabular}{|c|c|c|c|c|c|c|c|c|c|c|c|c|c|c|c|c|}
\hline YEAR & 1960 & 1961 & 1962 & 1963 & 1964 & 1965 & 1966 & 1967 & 1968 & 1969 & 1970 & 1971 & 1972 & 1973 & 1974 & 1975 \\
\hline Q1 & 354.40 & 345.00 & 355.40 & 384.50 & 385.20 & 388.20 & 355.60 & 303.00 & 298.90 & 339.80 & 423.30 & 444.60 & 420.30 & 410.70 & 847.00 & 1773.30 \\
\hline Q2 & 415.30 & 403.50 & 416.80 & 451.00 & 449.80 & 452.80 & 411.70 & 353.10 & 348.00 & 397.60 & 491.30 & 516.60 & 485.00 & 471.80 & 957.30 & 2001.00 \\
\hline Q3 & 433.60 & 420.50 & 435.40 & 471.40 & 467.90 & 470.30 & 424.60 & 366.00 & 360.30 & 413.80 & 507.20 & 533.80 & 497.60 & 481.90 & 961.70 & 2003.10 \\
\hline Q4 & 396.50 & 384.80 & 398.20 & 430.90 & 428.50 & 430.90 & 389.90 & 335.90 & 330.80 & 379.20 & 465.90 & 490.30 & 458.20 & 444.40 & 892.30 & 1861.90 \\
\hline
\end{tabular}

\begin{tabular}{|c|c|c|c|c|c|c|c|c|c|c|c|c|c|c|c|c|}
\hline YEAR & 1976 & 1977 & 1978 & 1979 & 1980 & 1981 & 1982 & 1983 & 1984 & 1985 & 1986 & 1987 & 1988 & 1989 & 1990 & 1991 \\
\hline Q1 & 1571.10 & 1698.10 & 1554.20 & 1425.30 & 1530.40 & 12952.50 & 13285.70 & 13257.80 & 12545.20 & 14556.40 & 15909.20 & 15375.30 & 16928.20 & 17846.20 & 18608.00 & 19267.20 \\
\hline Q2 & 1787.10 & 1933.80 & 1756.70 & 1584.30 & 1706.00 & 15080.20 & 15461.70 & 15360.50 & 14550.30 & 17069.10 & 18716.00 & 18063.80 & 19914.90 & 20989.80 & 21889.00 & 22701.70 \\
\hline Q3 & 1807.40 & 1958.00 & 1764.70 & 1563.90 & 1690.00 & 15618.00 & 16005.40 & 15831.70 & 15019.90 & 17813.20 & 19590.50 & 18887.70 & 20846.20 & 21949.90 & 22893.10 & 23779.60 \\
\hline Q4 & 1672.90 & 1811.80 & 1637.40 & 1459.90 & 1575.50 & 14338.90 & 14698.00 & 14559.60 & 13802.70 & 16309.70 & 17919.50 & 17281.20 & 19067.20 & 20092.20 & 20945.00 & 21755.10 \\
\hline
\end{tabular}

\begin{tabular}{|c|c|c|c|c|c|c|c|c|c|c|c|c|c|c|c|c|c|}
\hline YEAR & 1992 & 1993 & 1994 & 1995 & 1996 & 1997 & 1998 & 1999 & 2000 & 2001 & 2002 & 2003 & 2004 & 2005 & 2006 & 2007 & 2008 \\
\hline Q1 & 19628.00 & 19826.50 & 20287.60 & 21041.30 & 21933.00 & 22872.50 & 23825.20 & 25086.90 & 25821.60 & 26828.30 & 40887.10 & 43693.00 & 39973.80 & 42709.30 & 58433.00 & 62103.30 & 65692.10 \\
\hline Q2 & 23170.20 & 23478.30 & 24052.00 & 24932.60 & 25972.50 & 27086.60 & 28204.10 & 29673.90 & 30571.30 & 31759.10 & 49140.40 & 52562.30 & 50820.70 & 54385.30 & 74430.80 & 79378.10 & 83846.10 \\
\hline Q3 & 24316.20 & 24718.70 & 25351.60 & 26256.80 & 27339.90 & 28512.90 & 29675.50 & 31242.90 & 32166.00 & 33409.10 & 52424.30 & 56122.80 & 64795.10 & 69379.50 & 69953.50 & 76417.00 & 82856.60 \\
\hline Q4 & 22231.10 & 22573.00 & 23141.80 & 23981.00 & 24969.70 & 26042.00 & 27109.20 & 28543.60 & 29386.20 & 30525.10 & 47681.50 & 51031.70 & 60619.00 & 45781.80 & 48578.80 & 51516.60 & 94629.43 \\
\hline
\end{tabular}


